\title{
Semiconductor Split-Ring Resonators for Thermally Tunable, Terahertz Metamaterials
}

\author{
Jiaguang $\operatorname{Han}^{\mathrm{a}}$ \\ Department of Physics, National University of Singapore, 2 Science Drive 3, Singapore \\ 117542, Singapore
}

\begin{abstract}
Akhlesh Lakhtakiab
NanoMM-Nanoengineered Metamaterials Group, Department of Engineering Science and Mechanics, Pennsylvania State University, University Park, PA 16802, USA
\end{abstract}

\begin{abstract}
As the variation of temperature alters the intrinsic carrier density in a semiconductor, numerical simulations indicate that the consequent variation of the relative permittivity in the terahertz regime provides a way to realize thermally tunable split-ring resonators. Electromagnetic metasurfaces and metamaterials that are thermally tunable in the terahertz regime can thus be implemented.
\end{abstract}

Keywords: InSb, metamaterial, metasurface, semiconductor, split-ring resonator, terahertz, thermal tunability

\footnotetext{
${ }^{a}$ E-mail Address: phyhanj@nus.edu.sg

${ }^{\mathrm{b}}$ Corresponding Author. E-mail Address: akhlesh@psu.edu
} 


\section{Introduction}

Metamaterials are composite materials designed to exhibit an optimized combination of two or more responses to specific excitation. Typically, this multifunctionality is uncommon if not impossible in nature, and emerges from the engineered cellular morphology of a metamaterial. In other words, a metamaterial is an engineered assemblage of different types of mutually inert or mutually reinforcing cells of sufficiently small dimensions [1].

Among electromagnetic metamaterials, those that refract negatively have drawn considerable attention during this decade [2-4]. A type of cell used commonly in negatively refracting electromagnetic metamaterials is the planar splint-ring resonator (SRR), which is a two-dimensional metallic structure printed on a circuit-board substrate. A planar SRR is a resonant structure. The scope of a metamaterial containing tunable planar SRRs as some of its constituent cells is obviously very high and encompasses, for example, spatial light modulators and tunable optical filters.

Tunability strategies examined thus far include electrical control [5-7], magnetostatic control [8,9], and optical pumping [10]. Whereas the planar SRRs remain metallic in the cited publications, tunability is introduced by ensuring that another component (e.g., the substrate on which the SRRs are printed or another type of inclusions) is made of an electro-optic material, liquid crystal, ferrite, etc.

In this communication, we theoretically demonstrate the feasibility of metamaterials that are tunable in the terahertz regime by change of temperature. These metamaterials would not comprise metallic SRRs, but semiconductor SRRs instead. In the terahertz regime, semiconductors emulate metals by having the real parts of their 
relative permittivities negative. Compared to metals, however, semiconductors have a significant advantage in that their relative permittivity can be modified by changing the temperature, thereby changing the carrier density and, therefore, the plasma frequency of the semiconductor.

\section{Preliminaries}

For the sake of definiteness, let us consider a planar SRR made of InSb printed on an isotropic quartz substrate. Fig. 1a shows the schematic of a single SRR with linear dimensions in the plane ranging from 2 to $36 \mu \mathrm{m}$, and of thickness $200 \mathrm{~nm}$. The quartz substrate of relative permittivity 3.78 is $640-\mu \mathrm{m}$ thick. The essence of the chosen type of metamaterial is a metasurface [11]: a square array of InSb SRRs with lattice parameter 50 $\mu \mathrm{m}$, as shown in Fig. 1b. Computer simulations of the spectral response of this metasurface were performed using the commercial software CST Microwave Studio TM 2006B, which is a three-dimensional, full-wave solver employing the finite integration technique.

In the terahertz regime, the complex-valued relative permittivity of $\mathrm{InSb}$ is given by the simple Drude model [12]

$$
\varepsilon(\omega)=\varepsilon_{\infty}-\omega_{p}^{2} /\left(\omega^{2}+i \gamma \omega\right),
$$

where $\omega$ is the angular frequency; $\varepsilon_{\infty}$ represents the high-frequency value; $\gamma$ is the

damping constant; and the plasma frequency $\omega_{p}=\sqrt{N e^{2} / \varepsilon_{0} m^{*}}$ depends on the intrinsic carrier density $N$, the effective mass $m^{*}$ of free carriers, the electronic charge $e$, and the free-space permittivity $\varepsilon_{0}$. Compared to metals, the plasma frequency $\omega_{p}$ of $\mathrm{InSb}$ 
depends strongly on the temperature $T$. The intrinsic carrier density $N\left(\right.$ in $\left.\mathrm{cm}^{-3}\right)$ in $\mathrm{InSb}$ obeys the relationship [13]

$$
N=5.76 \times 10^{14} T^{3 / 2} \exp \left(-0.26 / 2 k_{B} T\right),
$$

where $k_{B}$ is the Boltzmann constant and the temperature is in Kelvin. A variation in $N$ due to a variation in $T$ thus changes $\omega_{p}$. Consequently, in the far-infrared portion of the terahertz regime, $\varepsilon(\omega)$ of $\mathrm{InSb}$ is very sensitive to $T$. Hence, for metasurfaces and metamaterials comprising InSb SRRs, we can expect that temperature variations can cause substantial variations in the optical response characteristics.
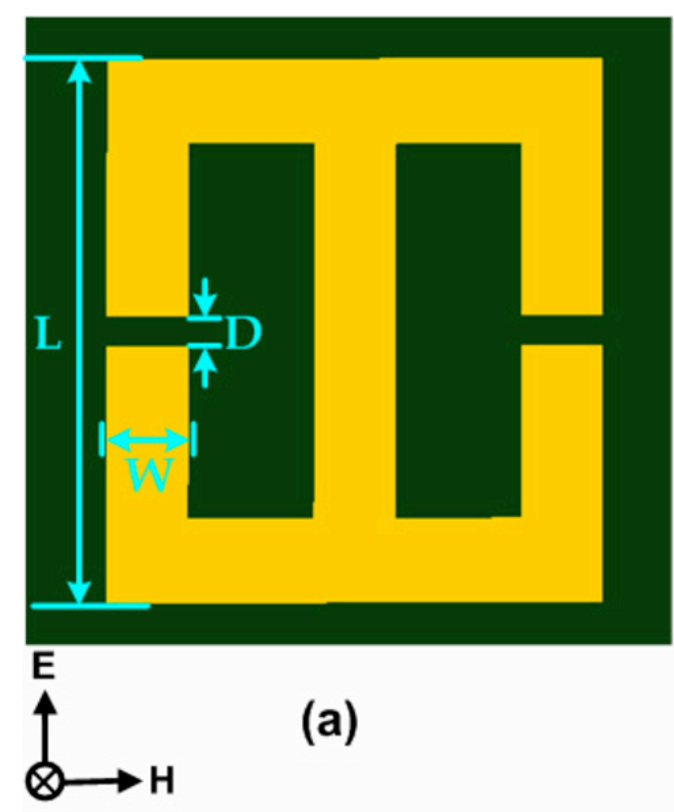

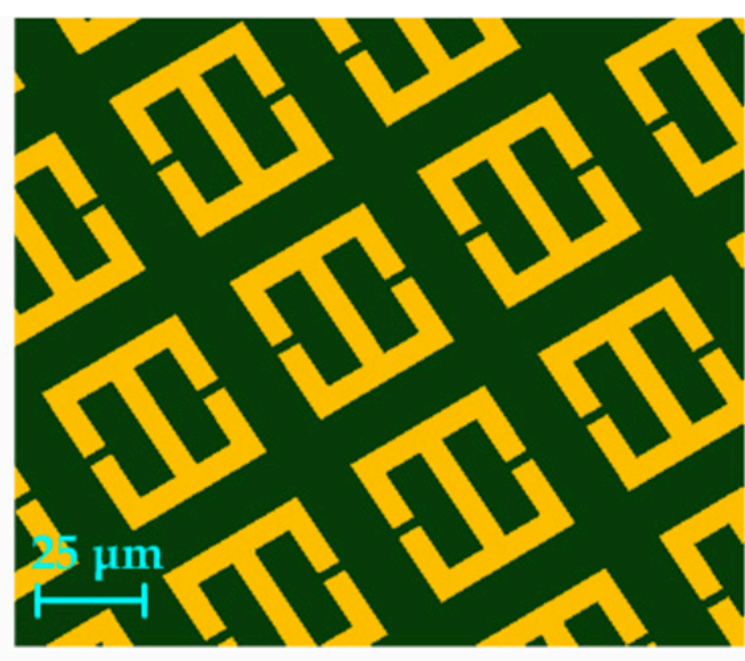

(b)

FIG. 1. (a) A single SRR with dimensions: $\mathrm{L}=36 \mu \mathrm{m}, \mathrm{D}=2 \mu \mathrm{m}$ and $\mathrm{W}=6 \mu \mathrm{m}$. (b) Schematic of a square array of SRRs printed on a quartz substrate with a period of $50 \mu \mathrm{m}$. The SRRs are made of InSb, whose relative permittivity is given by Eq. 1 with $\varepsilon_{\infty}=15.68$, $m^{*}=0.015 m_{e}, m_{e}=9.1 \times 10^{-31} \mathrm{~kg}$, and $\gamma / 2 \pi=0.05 \mathrm{THz}[12,13]$. The intrinsic free carrier concentration in InSb is [13]: $N=5.76 \times 10^{14} T^{3 / 2} \exp \left(-0.26 / 2 k_{B} T\right) \mathrm{cm}^{-3}$. The electric field of the normally incident plane wave is oriented perpendicular to the gaps in the SRRs. 
Suppose the chosen metasurface lies parallel to the plane $z=0$ of a Cartesian coordinate system such that the gaps in all SRRs are aligned parallel to the $x$ axis. Let the metasurface be illuminated by a plane wave whose wave vector is oriented parallel to the $z$ axis and whose electric field vector is aligned parallel to the $y$ axis. Using CST Microwave Studio TM 2600B, we computed the transmittance spectrum of the metasurface at frequencies below $1.0 \mathrm{THz}$ at various temperatures.

\section{Numerical Results and Discussion}

Figure $2 \mathrm{a}$ shows the computed frequency-dependent transmission of the chosen metasurface at various temperatures. The transmission is the ratio of the magnitude of the electric field of the transmitted plane wave to that of the incident plane wave. No remarkable resonance is evident at temperatures below $200 \mathrm{~K}$, and the transmission is almost independent of frequency at $1.0 \mathrm{THz}$ and lower frequencies. If the temperature is increased to $220 \mathrm{~K}$, a resonance is manifested as a small transmission dip around 0.09 THz. At $250 \mathrm{~K}$, the resonance becomes more visible and occurs at $0.15 \mathrm{THz}$ with a transmission minimum of 0.792 . The resonance blueshifts to $0.28 \mathrm{THz}$ with a transmission minimum of 0.549 at $300 \mathrm{~K}$, and then further to $0.44 \mathrm{THz}$ with a transmission minimum of 0.32 at $350 \mathrm{~K}$. Clearly, the transmission characteristics of the metasurface change significantly with temperature.

Figure $2 \mathrm{~b}$ shows a $3 \mathrm{D}$ profile of the resonant frequency corresponding with value of transmission dip as a function of temperature above $250 \mathrm{~K}$, for which purpose detailed simulations were performed at a temperature interval of $10 \mathrm{~K}$. As expected, the resonance 
of the metasurface can be significantly enhanced and blueshifted by raising the temperature.

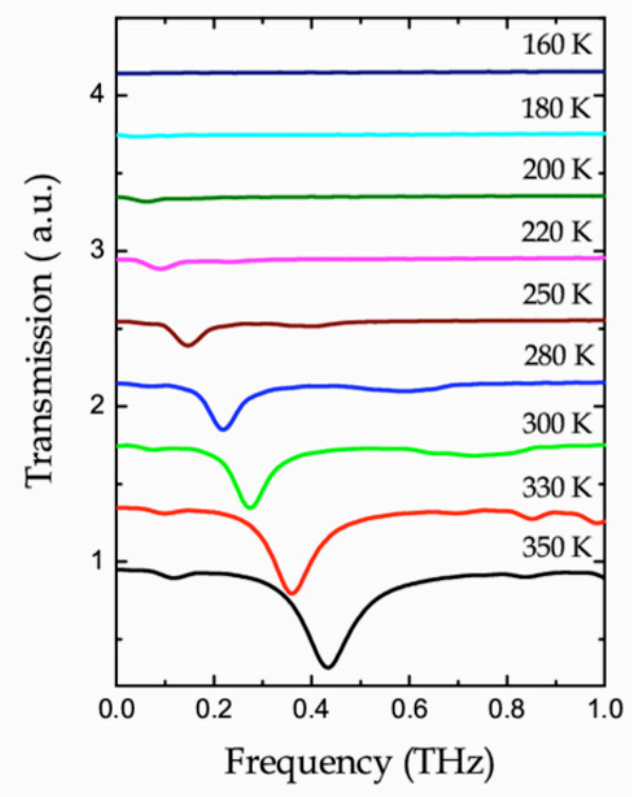

(a)

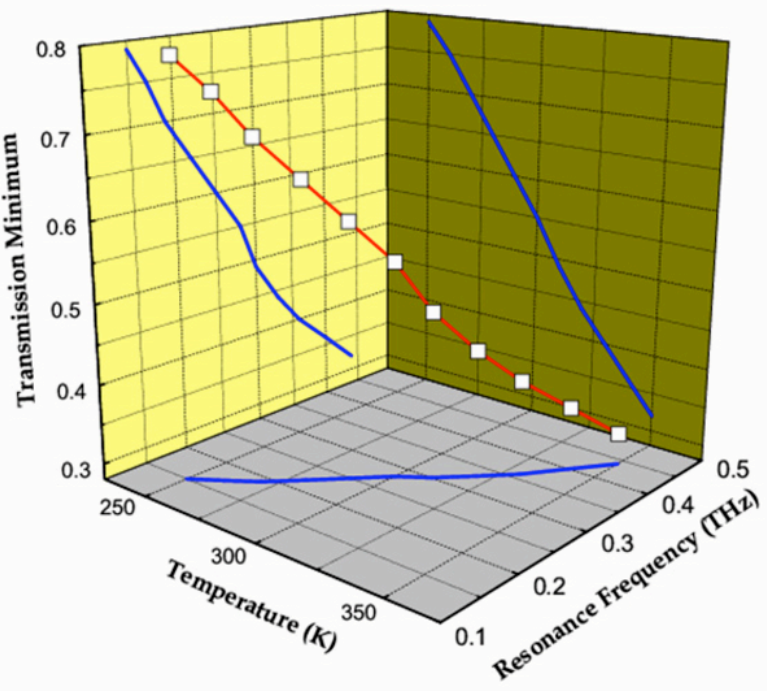

(b)

FIG. 2. (a) Transmission spectra of the chosen metasurface at different temperatures ranging from 160 to $350 \mathrm{~K}$. (b) Variation of the remarkable transmission minimum and the resonance with temperature above $250 \mathrm{~K}$.

The temperature-dependent resonant characteristics can be attributed directly to the increase in the density of free carriers by thermal excitation as the temperature increases. For instance, the intrinsic carrier density in InSb is $N \sim 0.94 \times 10^{14} \mathrm{~cm}^{-3}$ at 160 $\mathrm{K}$, but $N \sim 5.07 \times 10^{16} \mathrm{~cm}^{-3}$ at $350 \mathrm{~K}$; thus, the semiconductor displays more metallic features with increasing temperature.

This effect also can be seen from the variation of $\varepsilon(\omega)$ with $T$. The real part $\varepsilon_{r}(\omega)$ and the imaginary part $\varepsilon_{i}(\omega)$ of $\varepsilon(\omega)$ alter dramatically because of the variation of $N$ with $T$. At a specific value of $\omega, \varepsilon_{r}(\omega)$ reduces and $\varepsilon_{i}(\omega)$ increases as $T$ increases, as 
evinced by the plots in Fig. 3a. The more metallic behavior is also demonstrated by an increasing positive ratio $-\varepsilon_{r}(\omega) / \varepsilon_{i}(\omega)$ with temperature in Fig. $3 b$. Clearly, the InSb SRRs have almost insulating features at low temperatures but metallic features at high temperatures.

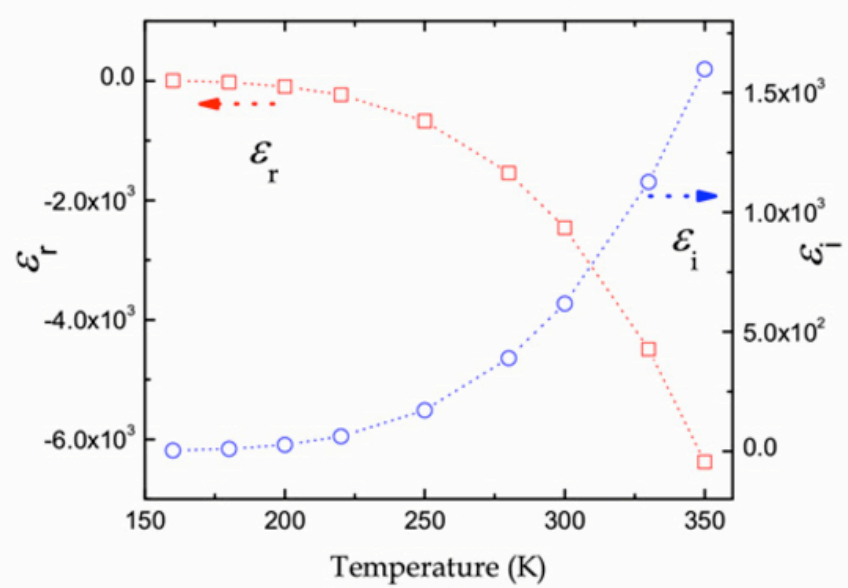

(a)

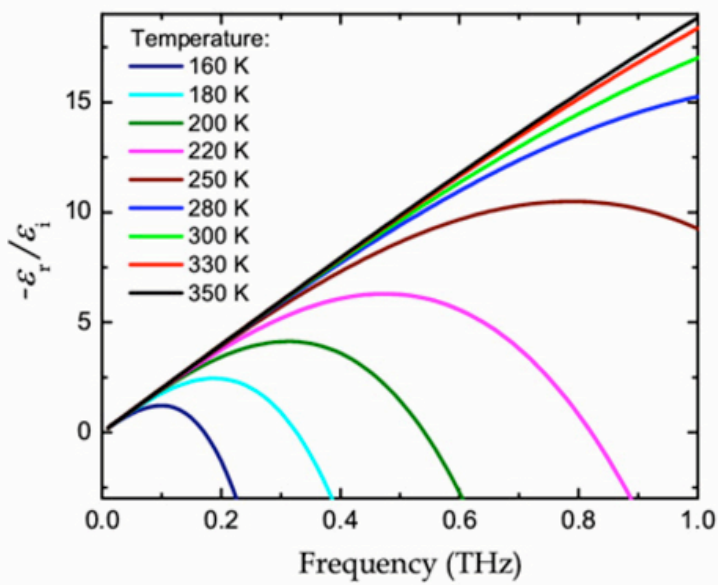

(b)

FIG. 3. (a) The real part $\varepsilon_{r}(\omega)$ (open squares) and the imaginary part $\varepsilon_{i}(\omega$ (open circles) of the relative permittivity of InSb at $0.2 \mathrm{THz}$ as functions of temperature. (b) Frequencydependent ratio $-\varepsilon_{r}(\omega) / \varepsilon_{i}(\omega)$ of InSb in the terahertz regime at different temperatures.

The resonance frequency turns out to be almost linearly related to $-\varepsilon_{r} / \varepsilon_{i}$, as shown in Fig. 4a. The enhancement of the resonance feature by increase of temperature is increased is further evident from the consequent increase of the $\mathrm{Q}$ factor, as presented in Fig. 4b. This increase of $Q$ with $T$ is ascribed to the better metallic characteristics of $\operatorname{InSb}$ at a higher temperature, and this conclusion is consistent with the recent experimental observation that an SRR made of a better metal has a more pronounced resonance [14]. 


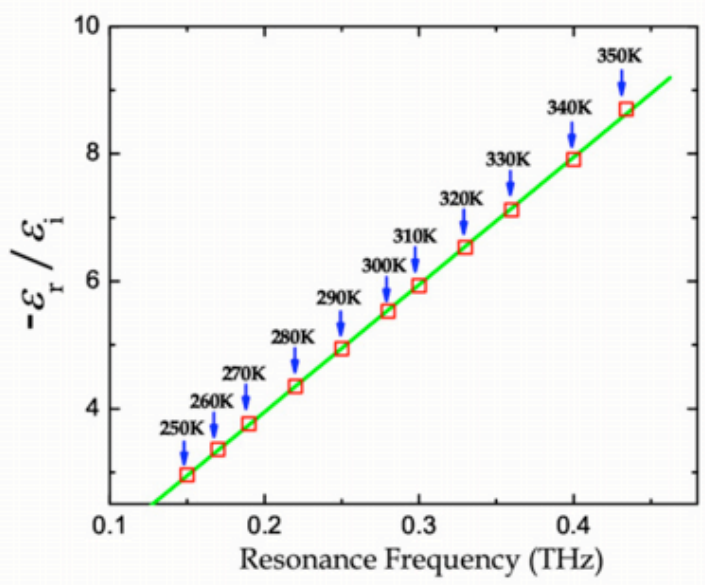

(a)

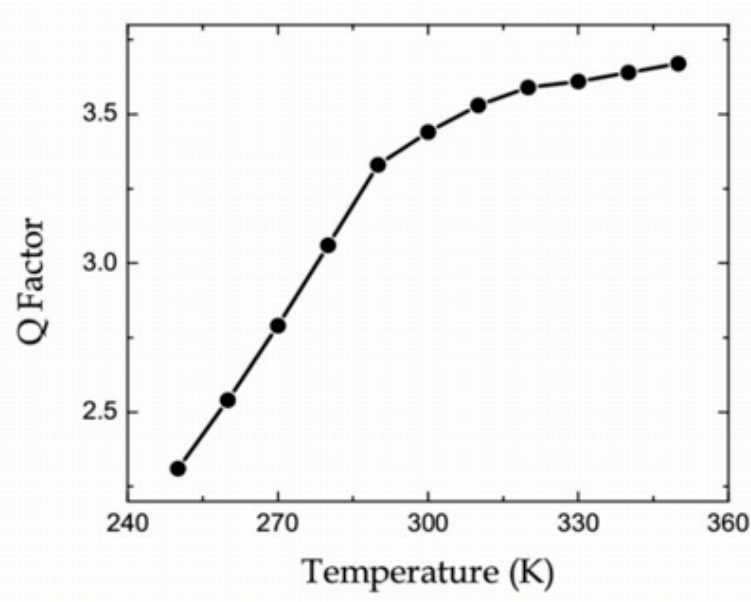

(b)

FIG. 4. (a) The ratio $-\varepsilon_{r}(\omega) / \varepsilon_{i}(\omega)$ at the resonance frequency at different temperatures. The solid line indicates a good linear fit. (b) The quality factor $Q$ of the transmission dip at different temperatures.

\section{Concluding Remarks}

So we have shown that as a variation of temperature alters the intrinsic carrier density in a semiconductor, the consequent variation of the relative permittivity in the terahertz regime provides a way to make thermally tunable split-ring resonators and even other types of cells for use in assembling electromagnetic metamaterials. These meteametarials would therefore be thermally tunable in the terahertz regime. Furthermore, any other mechanism for varying the intrinsic carrier density would endow tunability to electromagnetic metamaterials comprising semiconducting split-ring resonators, etc.

Acknowledgements. JH acknowledges fruitful discussions with Prof. Takeda and Dr. Miyamaru (Shinshu University, Japan) and financial support from the MOE Academic Research Fund of Singapore. 


\section{References}

[1] Walser, R.M. in: Weiglhofer, W.S.; Lakhtakia, A. (Eds.) Introduction to Complex Mediums for Electromagnetics and Optics; SPIE Press: Bellingham, WA, USA, 2003.

[2] Lakhtakia, A.; McCall, M.W.; Weiglhofer, W.S. AEÜ Int. J. Electron. Commun. 2002, 56, 407-410.

[3] Ramakrishna, S.A. Rep. Prog. Phys. 2005, 68, 449-521.

[4] Eleftheriades, G.V.; Balmain, K.G. (Eds.) Negative Refraction Metamaterials: Fundamental Principles and Applications; Wiley: Hoboken, NJ, USA, 2005.

[5] Chen, H.-T.; Padilla, W.J.; Zide, J.M.O.; Gossard, A.C.; Taylor, A.J.; Averitt, R.D. Nature 2006, 444, 579-600.

[6] Zhao, Q.; Kang, L.; Du, B.; Li, B.; Zhou, J.; Tang, H.; Liang, X.; Zhang, B. Appl. Phys. Lett. 2007, 90, 011112.

[7] Hand, T.H.; Cummer, S.A. J. Appl. Phys. 2008, 103, 066105.

[8] He, Y.; He, P.; Harris, V.G.; Vittoria, C. IEEE Trans. Magn. 2006, 42, 2852-2854.

[9] Kang, L.; Zhao, Q.; Zhao, H.; Zhou, J. Opt. Express 2008, 16, 8825-8834.

[10] Degiron, A.; Mock, J.J.; Smith, D.R. Opt. Express 2007, 15, 1115-1127.

[11] Lapine, M.; Tretyakov, S.A. IET Microw. Antennas Propag. 2007, 1, 3-11.

[12] Howells, S.C.; Schlie, L.A. Appl. Phys. Lett. 1996, 69, 550-552.

[13] Halevi, P.; Ramos-Mendieta, F. Phys. Rev. Lett. 2000, 85, 1875-1878.

[14] Singh, R.; Azad, A.K.; O’Hara, J.F.; Taylor, A.J.; Zhang, W.L. Opt. Lett. 2008, 33, 1506-1508. 